\title{
Inflammasome formation in the lungs of patients with fatal COVID-19
}

\author{
Stefano Toldo ${ }^{1} \cdot$ Rossana Bussani $^{2} \cdot$ Vincenzo Nuzzi $^{3} \cdot$ Aldo Bonaventura $^{1} \cdot$ Adolfo G. Mauro $^{1} \cdot$ Antonio Cannatà $^{3}$. \\ Raghavendra Pillappa ${ }^{4} \cdot$ Gianfranco Sinagra $^{4} \cdot$ Patrick Nana-Sinkam $^{5} \cdot$ Patricia Sime $^{5} \cdot$ Antonio Abbate $^{1}$ (1)
}

Received: 13 August 2020 / Revised: 1 October 2020 / Accepted: 12 October 2020 / Published online: 20 October 2020

(c) Springer Nature Switzerland AG 2020

\begin{abstract}
Objective The orf8b protein of the coronavirus SARS-CoV, analogous to SARS-CoV-2, triggers the NLRP3 inflammasome in macrophages in vitro. Deregulated inflammasome-mediated release of interleukin-1 family cytokines is important in hyper-inflammatory syndromes, like happens in SARS-CoV-2-mediated cytokine release syndrome. We propose that an intense inflammasome formation characterizes the lungs of patients with fatal COVID-19 disease due to pneumonia and acute respiratory distress syndrome (ARDS).

Methods Samples from four patients with confirmed COVID-19 pneumonia who had been hospitalized at the Hospital of the University of Trieste (Italy) and died of ARDS and four lung samples from a historical repository from subjects who had died of cardiopulmonary arrest and had not been placed on mechanical ventilation and without evidence of pulmonary infection at postmortem examination were collected. Pathology samples had been fixed in formalin $10 \%$ at time of collection and subsequently embedded in paraffin. We conducted staining for ASC (Apoptosis-associated Speck-like protein containing a Caspase recruitment domain), NLRP3 (NACHT, LRR, and PYD domains-containing protein 3), and cleaved caspase-1.

Results Intense expression of the inflammasome was detected, mainly in leukocytes, within the lungs of all patients with fatal COVID-19 in the areas of lung injury. The number of ASC inflammasome specks per high power fields was significantly higher in the lungs of patients with fatal COVID-19 as compared with the lungs of control subjects ( $52 \pm 22$ vs $6 \pm 3$, $P=0.0064)$.

Conclusions These findings identify the presence of NLRP3 inflammasome aggregates in the lungs of fatal COVID-19 pneumonia thus providing the potential molecular link between viral infection and cytokine release syndrome.
\end{abstract}

Keywords SARS-CoV-2 $\cdot$ COVID-19 $\cdot$ NLRP3 inflammasome $\cdot$ IL- 1 Cytokine release syndrome

Responsible Editor: John Di Battista.

Stefano Toldo and Rossana Bussani equally contributed to the realization of this manuscript.

Electronic supplementary material The online version of this article (https://doi.org/10.1007/s00011-020-01413-2) contains supplementary material, which is available to authorized users.

Stefano Toldo

stefano.toldo@vcuhealth.org

1 VCU Pauley Heart Center, Virginia Commonwealth University, 1220 E Broad St, Richmond, VA 23298, USA

2 Istituto di Anatomia Patologica, Università di Trieste, Trieste, Italy

3 Istitituto di Cardiologia, Università di Trieste, Trieste, Italy

4 Anatomic Pathology, Virginia Commonwealth University, Richmond, VA, USA

5 Division of Pulmonary and Critical Care Medicine, Virginia Commonwealth University, Richmond, VA, USA

\section{Introduction}

The severe acute respiratory syndrome coronavirus 2 (SARS-CoV-2) is responsible for a clinical syndrome in humans referred to as coronavirus disease 2019 (COVID19) that ranges from mild self-limiting rhinopharyngitis to severe acute respiratory distress syndrome (ARDS) which can be fatal [1]. The mechanisms by which ARDS ensues are incompletely understood, but is considered to be related to an inappropriate release of pro-inflammatory cytokines [2]. Elevated levels of pro-inflammatory mediators predict worse outcomes and identify patients in the hyper-inflammatory stage $[1,2]$, hence the need to better understand the inflammatory response.

The orf8b protein of the coronavirus SARS-CoV, analogous to SARS-CoV-2, triggers the NLRP3 (NACHT, LRR, and PYD domains-containing protein 3 ), the sensor protein 
of the intracellular macromolecular structure-the inflammasome, in macrophages in vitro [3-5]. Deregulated inflammasome-mediated release of cytokines of the interleukin-1 (IL-1) family is central in hyper-inflammatory syndromes, thus representing a potential link between SARS-CoV-2 infection and cytokine release syndrome in COVID-19. Despite the interest in the inflammasome in COVID-19, there have been no reports identifying the inflammasome in human lung samples to date.

We propose that an intense inflammasome formation characterizes the lungs of patients with fatal COVID-19 disease due to ARDS.

\section{Methods}

To this aim, we obtained post-mortem samples from four patients (median age 71 years, range between 43 and 77, 3 [75\%] males) with SARS-CoV-2 infection confirmed by real-time reverse-transcriptase polymerase chain reaction from nasopharyngeal swabs who had been hospitalized at the Hospital of the University of Trieste and died of ARDS a median of 15 days after admission (range 1-29). All four patients had critical COVID-19 pneumonia with respiratory failure requiring mechanical ventilation for ARDS for a median duration of 7 days (range 1-28 days).

For comparison, we obtained lung samples from a historical repository from pre-COVID-19 era deriving from four subjects (median age 55, range between 51 and 57, 2 [50\%] males, 3 [75\%] smokers) who had died of cardiopulmonary arrest and had not been placed on mechanical ventilation and no evidence of pulmonary infection at postmortem examination.

All pathology samples had been fixed in formalin 10\% at time of collection and subsequently embedded in paraffin.

The samples were then cut in $4 \mu \mathrm{m}$ sections and underwent rehydration, antigen retrieval, and treated with a primary and secondary antibody directed against ASC (Apoptosis-associated Speck-like protein containing a Caspase recruitment domain), a scaffold protein for the inflammasome and appearing as a cytoplasmic speck as it aggregates, or against the NLRP3, the sensor protein in the inflammasome, or against cleaved caspase-1, the effector enzyme in the inflammasome that cleaves pro-IL- $1 \beta$ into active IL- $1 \beta$, as previously described $[6,7]$. Negative control experiments were conducted by omitting the primary or the secondary antibody or both.

Images were acquired using a IX70 microscope and Cellsense software (both Olympus, Center Valley, PA, USA) using a $40 \times$ objective $(400 \times$ magnification) and quantified by two investigators (ST, AA) unaware of group allocation by measuring the numbers of ASC-containing specks per high-power fields [6]. Morphology was used to identify to leukocytes and alveolar epithelial cells.

\section{Results}

Evidence of lung injury was present in all four cases of fatal COVID-19 cases and not in the control subjects. Intense expression of the inflammasome was detected, mainly in leukocytes, in the lungs of all patients with fatal COVID-19 in the areas of lung injury. A small number of inflammasome specks were found in leukocytes also in control subjects, but a lesser degree.

The number of ASC inflammasome specks per high power fields was significantly higher in the lungs of patients with fatal COVID-19 as compared with the lungs of control subjects ( $52 \pm 22$ vs $6 \pm 3, P=0.0064)$ (Fig. 1). A similar trend of significantly increased expression was observed for NLRP3 and caspase-1 in lungs from COVID-19 patients compared with control lungs (Supplementary Figure).

Representative images from patients with COVID-19 and control lungs are shown in Fig. 1: panel a shows staining for the scaffold protein, ASC; panel $\mathrm{b}$ shows staining for the sensor for viral proteins, NLRP3; and panel C shows staining for the effector enzyme of the inflammasome.

While preferential to leukocytes, the expression of ASC and caspase- 1 was evident also in alveolar epithelial cells.

\section{Discussion}

We herein report for the first time the presence of the inflammasome in the lungs of patients with fatal COVID-19. This provides, to the best of our knowledge, the first ex vivo evidence linking the SARS-CoV-2 to a mechanism of activation of inflammasome and release of pro-inflammatory cytokines that may be, at least in part, responsible for the injury to the lungs.

The SARS-CoV-2 virus enters the body through the airways and uses the angiotensin-converting enzyme 2 expressed on alveolar epithelial cells, macrophages, and endothelial cells [8]. As NLRP3 inflammasome is formed in response to a variety of infectious and non-infectious stimuli [5], NLRP3 inflammasome formation should not be in any way be considered unique to SARS-CoV-2 infection. Indeed, it is reasonable to expect inflammasome formation in forms of ARDS other than the one associated with COVID-19. The finding of the NLRP3 inflammasome in critical COVID-19 lung disease, however, adds to the literature as it is the first report to this point. In addition, it opens to the possibility that treatments directed against the NLRP3 inflammasome or derived cytokines, such as IL-1 $\beta$ and IL-18, may affect the lung pathology in 
Fig. 1 Inflammasome in the lungs of patients with fatal COVID-19 infection. Representative images of immunofluorescence from pathology specimens of patients with fatal COVID-19 pneumonia (panels a, c, and e) or subjects who died of cardiopulmonary arrest without evidence of pulmonary infection (panels $\mathbf{b}, \mathbf{d}$ and $\mathbf{f}$ ). Staining for inflammasome structures is identified in red in panels A-B, C-D and E-F for ASC (Apoptosis-associated Speck-like protein containing a Caspase recruitment domain), NLRP3 (NACHT, LRR, and PYD domains-containing protein 3), and caspase-1, respectively. Panel $\mathbf{g}$ illustrates the quantification of the presence of the inflammasome, expressed as ASC specks per high-power fields, showing a significantly higher number of inflammasomes in COVID-19 patients versus controls. Data expressed as mean and standard deviation. $P$ value generated using 2-tails $T$ test for unpaired samples
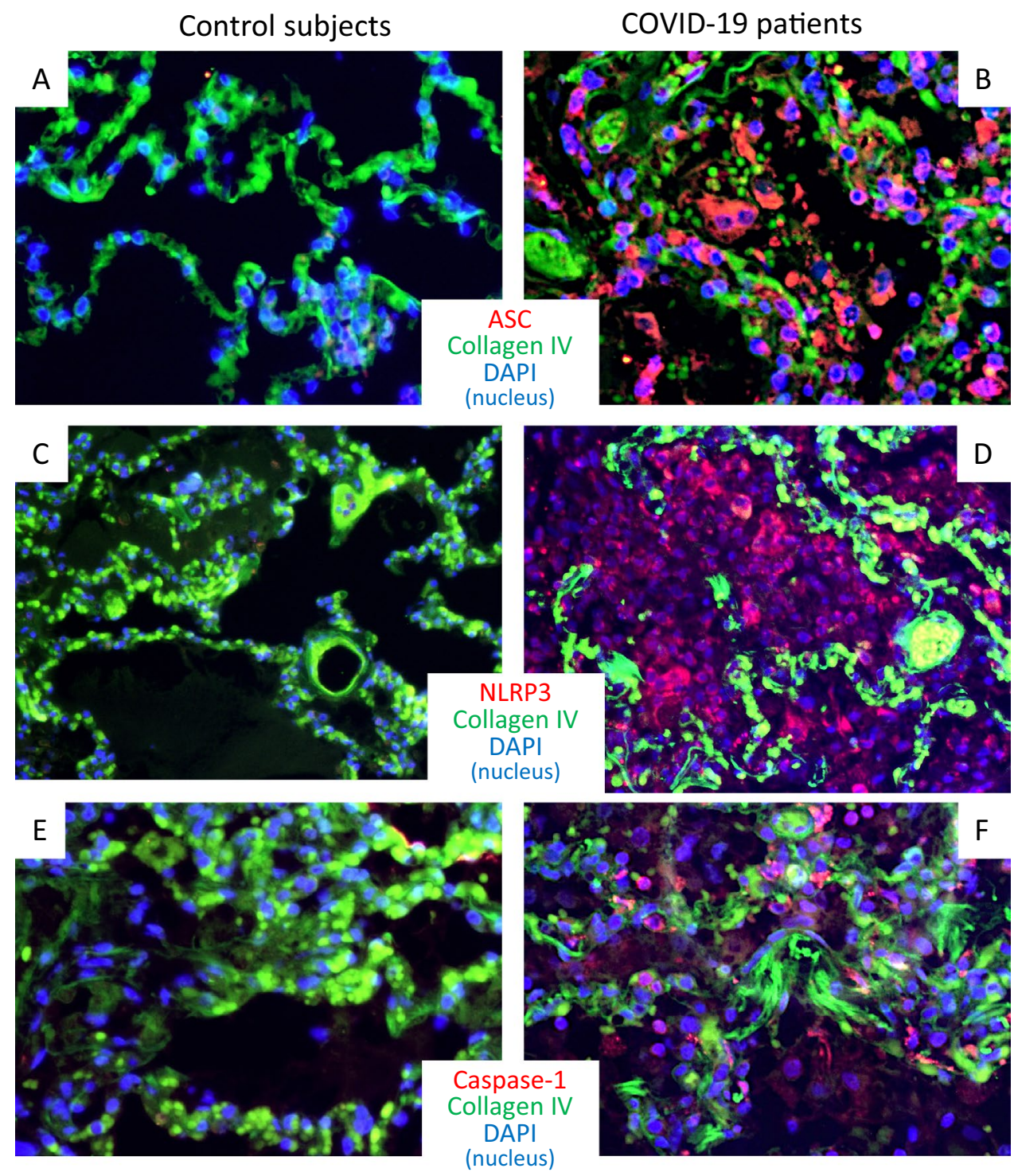

G

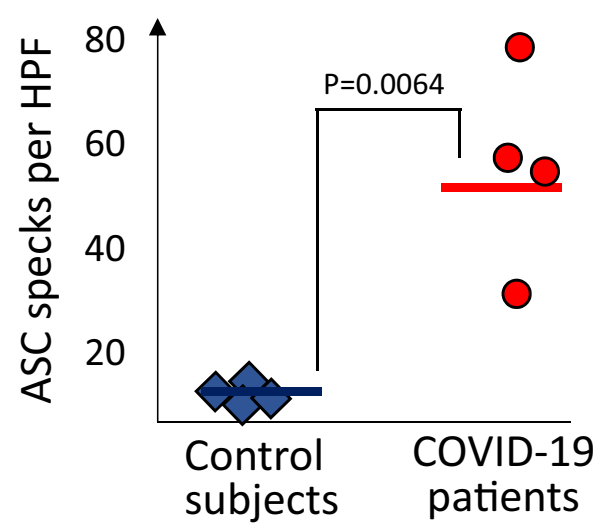

Additional pathology studies are also warranted with adequate number of samples available, to establish the time course of inflammasome formation, type of cell forming the inflammasome structure, co-localization with viral antigens
COVID-19 [9-12]. NLRP3 inflammasome inhibitors are also under development and being explored for the treatment of COVID-19 (NCT04540120). These hypotheses would, however, need to be tested in clinical trials. 
and viral clearance, and evolution toward organizing pneumonia [13].

In conclusion, although limited by the small number of observations, the data presented herein identify the presence of NLRP3 inflammasome aggregates in the lungs of fatal COVID-19 patients, thus providing the potential molecular link between viral infection and cytokine release syndrome.

Author contributions Study ideation and design: ST, RB, AC, AA. Sample collection and preparation: ST, RB, RP, PN-S, PS. Sample processing and experimentation: ST, RB, AB, AGM, RP. Data acquisition and measurements: ST, AA, RP, VN. Statistical analysis: ST, AA. Manuscript-initial draft: ST, AA. Manuscript—critical revision and approval: RB, VN, AB, AGM, GS, RP, PN-S, PS.

Funding Dr. Abbate and Dr. Toldo are supported by a United States of America National Institute of Health NHLBI award (R01HL150115). The funding source had no direct role in and carry no responsibility for this study.

\section{Compliance with ethical standards}

Conflict of interest Dr. Toldo reports grants from Olatec, grants from Kiniksa during the conduct of the study. Dr. Bonaventura reports a travel award grant from Kiniksa, outside the submitted work. Dr. Abbate reports grants and personal fees from Olatec, grants from Novartis, grants and personal fees from Kiniksa, grants from Regeneron, during the conduct of the study. In addition, Dr. Abbate has a patent on a Cryopyrin inhibitor issued. All other authors report no conflicts relevant to this work.

Ethics committee approval The research was conducted on de-identified post-mortem samples; this research did not involve any human living subjects.

\section{References}

1. Guan WJ, Ni ZY, Hu Y, Liang WH, Ou CQ, He JX, et al. Clinical characteristics of coronavirus disease 2019 in China. N Engl J Med. 2020;382:1708-20.

2. Iannaccone G, Scacciavillani R, Del Buono MG, Camilli $\mathrm{M}$, Ronco C, Lavie CJ, et al. Weathering the cytokine storm in COVID-19: therapeutic implications. Cardiorenal Med. 2020;10:277-87.

3. Shi CS, Nabar NR, Huang NN, Kehrl JH. SARS-Coronavirus Open Reading Frame-8b triggers intracellular stress pathways and activates NLRP3 inflammasomes. Cell Death Discov. 2019;5:101.

4. Freeman TL, Swartz TH. Targeting the NLRP3 inflammasome in severe COVID-19. Front Immunol. 2020;11:1518.

5. Abbate A, Toldo S, Marchetti C, Kron J, Van Tassell BW, Dinarello CA. Interleukin-1 and the inflammasome as therapeutic targets in cardiovascular disease. Circ Res. 2020;126:1260-80.

6. Toldo S, Kannan H, Bussani R, Anzini M, Sonnino C, Sinagra G, et al. Formation of the inflammasome in acute myocarditis. Int $\mathrm{J}$ Cardiol. 2014;171:e119-21.

7. Kron J, Mauro AG, Bonaventura A, Toldo S, Salloum FN, Ellenbogen $\mathrm{KA}$, et al. Inflammasome formation in granulomas in cardiac sarcoidosis. Circ Arrhythm Electrophysiol. 2019;12:e007582.

8. Hoffmann M, Kleine-Weber H, Schroeder S, Kruger N, Herrler T, Erichsen S, et al. SARS-CoV-2 cell entry depends on ACE2 and TMPRSS 2 and is blocked by a clinically proven protease inhibitor. Cell. 2020;181(271-280):e8.

9. Cavalli G, De Luca G, Campochiaro C, Della-Torre E, Ripa M, Canetti D, et al. Interleukin-1 blockade with high-dose anakinra in patients with COVID-19, acute respiratory distress syndrome, and hyperinflammation: a retrospective cohort study. Lancet Rheumatol. 2020;2:e325-31.

10. Cauchois R, Koubi M, Delarbre D, Manet C, Carvelli J, Blasco VB, et al. Early IL-1 receptor blockade in severe inflammatory respiratory failure complicating COVID-19. Proc Natl Acad Sci USA. 2020;117:18951-3.

11. Ucciferri C, Auricchio A, Dinicola M, Potere N, Abbate A, Cipollone $\mathrm{F}$, et al. Canakinumab in a subgroup of patients with COVID19. Lancet Rheumatol. 2020;2:457-8.

12. Vecchie A, Bonaventura A, Toldo S, Dagna L, Dinarello CA, Abbate A. IL-18 and infections: is there a role for targeted therapies? J Cell Physiol. 2020. https://doi.org/10.1002/jcp.30008.

13. Sauter JL, Baine MK, Butnor KJ, Buonocore DJ, Chang JC, Jungbluth AA, et al. Insights into pathogenesis of fatal COVID-19 pneumonia from histopathology with immunohistochemical and viral RNA studies. Histopathology. 2020. https://doi.org/10.1111/ his. 14201

Publisher's Note Springer Nature remains neutral with regard to jurisdictional claims in published maps and institutional affiliations. 